\title{
Meaning Loss in Translating Commissive Speech Acts in Movie Subtitles from English into Kurdish
}

\author{
Muhammed Kamal Abdulwahab1, Himdad A. Muhammad2, Mary Beneditte ${ }^{3}$ \\ 1,2College of Basic Education, Salahaddin University, Kurdistan Region - Iraq \\ ${ }^{3}$ College of Education, Criminal Justice and Human Services, University of Cincinnati- USA
}

\begin{abstract}
The paper aims at investigating translating commissive speech acts in movie subtitle from English into Kurdish. It attempts to identify the meaning loss, in other words deletion errors and then categorize them along with the illocutionary acts. The samples of the study are taken from the subtitle of the horror movie (Pumpkinhead: Blood Feud) which has been translated into the Kur dish language. First, twenty commissive speech act are identified as having translation problems in the movie according to Searle's category, then their illocutionary acts are specified. The English and Kurdish subtitles are compared to find out the meaning loss and deleted items, in addition to the compatibility of the illocutionary acts between them. The results of the analysis show that 'promising' and 'warning' are the most challenging illocutionary acts for translators because of the number of deletion errors found in their translation. The most common type of meaning loss in the translation of commissive speech acts are adverbs, pronouns, exclamation words, main verbs, modal verbs, nouns, and adjectives, respectively.
\end{abstract}

Keywords: Subtitle, Speech acts, meaning loss.

\section{Introduction}

Translation studies are now regarded as an independent academic discipline. This discipline is important in various fields. The development of knowledge, trade, and the impact of globalization have caused a revolution in the translation field. According to Colina (2015), translation is 'a process of product of transforming written texts from one human language into another. It generally requires a necessary degree of resemblance to or correspondence with the source text'. As for the types of translation, several classifications based on function, meaning, and level have been introduced. Human, mechanical and, computer-aided are among the most common types and each of these has several subtypes and modes.

Subtitling is regarded as a mode of audio-visual translation which has become very common in the last few decades due to the dominance of movie production, especially in Hollywood and Bollywood (Rao:2007 \& Crane:2014). Luyken et al. (1991) define a subtitle in the sense that the original dialogue is condensed in written translation and showed as lines of text usually located at the foot of the screen. It appears and disappears to coincide in time with the corresponding oral dialogue and, it is inserted into the screen as the final stage of post production activity.

The increasing demand on movie translation from countries of different languages has paved the way to the development of subtitling. According to Gottlieb (2005), subtitle is a prepared communication employing written language, acting as a synchronous channel and added as a part of polysemiotic text. The subtitles are derived from the spoken utterances of the movie so the mode changes from spoken to written. In addition to that, the differences between the languages in question, it can be said that errors are inevitable in the translation process. The errors are of different kinds, such as mistranslation, deletion, addition, and foreign translation. This study focuses on deletion errors only. In movie scripts, like any other type of language, there are various aspects that can be independently investigated .The current study is concerned with Speech Acts in general, commissive speech acts in particular, within movie subtitles. Speech acts are studied within the context of subtitling. According to 
Fromkin et al. (2003), speech act is the action or intent that a speaker accomplishes when using language in context, the meaning of which is inferred by hearers.

This study aims at firstly, investigating the existence of meaning loss in translating commissive speech acts and their illocution in movie subtitle (Pumpkinhead: Blood Feud). It also attempts to find out to what extent Searle's speech acts and their illocutionary acts in English language are compatible with those in the Kurdish language. Finally, it explores the types of deletion error, which are committed in the translation of commissive speech acts.

\section{Theoretical Background}

\subsection{Background}

Translation is regarded as an activity of mediating meaning from source language into target language. The existence of semantic, pragmatic and cultural differences among languages makes the translators' task very hard (Liu: 2003 \& Guerra, 2012). Muhammed (2017) Emphasizes the existence of such differences between English and Kurdish. Hence, the process of translation is bound to face many problems. According to Ghazala (2008) translators may face various types of problems as follows:

- Grammatical problems: These are the product of the source language complicated grammar as well as grammatical and structural differences between both languages.

- Lexical problems: These are faced in the translation of, for example, synonymy, polysemy, monosemy, collocations, idioms, proverbs, metaphors, Technical translation: Arabization, proper names, titles, political establishments, geographical terms and, UN acronyms.

- Stylistic problems: These are found in the translation of formal vs. informal style, fronting, parallelism, ambiguity, complex vs. simple style, long sentences vs. short style, passive vs. active style, repetition and variation, redundancy, the style of the show of muscle, normalization vs. verbalization and The style of irony.

- Phonological problems: These deal with sounds and their effect on meaning.

To sum up, it can be seen that translation errors are of various kinds. This study deals with some grammatical and lexical problems within subtitle translation, such as the deletion of some items.

\subsection{Speech Acts}

Fromkin et al. (2003) define a speech act as the action or intent that a speaker accomplishes when using language in context, the meaning of which is inferred by hearers. According to LoCastro (2012) and Al Sulaimaan (2010), philosophers have drawn a distinction between speaking and action. However, Austin claims that utterances are equivalent to actions, such as (I now pronounce you man and wife) where the utterance creates a new social reality.

There are several types of speech acts in the movie scripts. This study depends on Searle's category of speech acts. Searle cited in Leech (1983), identifies five types of speech acts, namely declaratives, expressives, representatives, commissives, and directives. This study concentrates on commissive speech acts only. Searle as cited in Yule (1996), explains that commissive speech acts, commit the speaker to future course of actions (I am going to get it right next time), the illocutions might be promising, warning, refusing, and guaranteeing.

\subsection{Literature Review}

There are a lot of studies concerning the classification of speech acts in movie subtitles, but there are few studies concerning translating such acts in movie subtitles. One of the previous studies was done by Muhammad (2017) who conducted a study entitled (Semantic loss in Translating Movie Subtitles from English into Kurdish (Witch Hunter as a Sample). The study dealt with cases of under-translation, over-translation, and mistranslation in subtitles as samples of semantic loss. 
The researcher depended on Baker's typology of equivalence, especially the equivalence and nonequivalence at the word level. The adopted approach of the study was descriptive qualitative and the content analysis type was employed. The research data have been taken from the English and Kurdish scripts of the English movie Witch Hunter. The main results of the study showed that the frequency of over translation is lower than the other losses due to the nature of subtitling constrains, such as the space and time factor. However, the frequency of under-translation is higher. This is because the time and space factor is encouraging or sometimes obliging the translator to omit some unnecessary words or some words that do not influence the message. The highest frequency is the mistranslation of various types, such as singular to plural or plural to singular, tense shift, definite to indefinite, equivalence choice, word order shift, structure shift, synonyms, and antonyms.

Another study by Abdulwahab et al (2020) was entitled (Translating Directive Speech Acts in Movie Subtitle from English into Kurdish). It dealt with four translation problems namely mistranslation, deletion, addition, and foreign translation in subtitle. The adopted approach of the study was descriptive qualitative and the content analysis type was employed. The research data were taken from the English and Kurdish scripts of the English Horrer movie (Pumpkinhead: Blood Feud). The results of the study showed that most speech acts and their illocutions in the English language were compatible with the translated ones in the Kurdish language. Whereas wide gap in translation between the original text and target text leads to incompatibility of speech acts and their illocutions in several cases. The most common type of error is mistranslation, within mistranslation phrasal verbs were the most challenging. Deletion is the second most common type of error, the most deleted items are adverbs. Addition is the third most common type of error, punctuation is the most common type of addition. Finally, foreign translation is the least common type of translation error.

Sultan (2007) who conducted a study entitled (The semantics, pragmatics and translation of speech acts) from English into Arabic language. The researcher employed functional equivalence. The adopted approach of Sultan study was qualitative approach. The research data were taken from Quranic verses. The results of the study showed that semantically there was no difference between English and Arabic speech acts. The realization of speech act in English language is gramatilized, however the speech act in Arabic language is lexicalized. In addition to that, both languages favor the use of indirect speech acts. Functional equivalence is the more appropriate type of equivalence for translating speech acts because it depends on rendering the functions of speech acts.

What makes this study different from the previous studies is not only that speech acts are covered in general but more specifically that the commissive speech acts and their illocutions are investigated. The second distinctive feature of this study is the investigation of Kurdish subtitling of English movies which is a relatively new area in the Kurdish community; hence there is a research gap.

\section{Methodology}

This study adopted a descriptive qualitative approach. According to Mackey and Gass (2005), a qualitative approach is like an inductive path that commences with few notions followed by narrowing in focus. The nature of the data requires content analysis of the qualitative approach so as to explore how commissive speech acts have been translated from English into Kurdish. This study depended on Dynamic Equivalence in which the target language should hold cultural expectation and linguistic needs in addition to having naturalness of expression. Nida (1964) cited in Munday (2012) mentions that naturalness is achieved through the selection of lexicon, grammar adjustment and cultural reference, this is also supported. The errors are highlighted in the tables. This symbol (=) refers to the 
compatibility of illocutions and this symbol (\#) refers to the incompatibility of illocutions between the English and the Kurdish language.

\section{Data Collection and Scope}

According to Mackey and Gass (2005), sampling is a strategy employed in choosing data or participants. The data of the study have been taken from a movie subtitle (Pumpkinhead: Blood Feud) broadcasted in 2007. This movie was translated into Kurdish after two years by a company. It has been selected due to the availability of movie scripts of both languages i.e., English and Kurdish. The Content analysis is employed in this research. According to Krippendorp (2004), content analysis is a procedure used to make valid and replicable inferences from a text to their context of usage. In line with this, Schreier (2012) defines qualitative content analysis as a technique implemented to describe the meaning of material systematically; it is applicable via classifying materials, such as coding. This study tries to investigate the frequency of commissive speech acts and their illocutions in comparison to the Kurdish language. Moreover, the study tries to identify the deletion error. In line with this, Anderson and Arsenault (1998) argue that content analysis has the ability to explain the relative frequency and significance of specific topics.

The scope of this study is restricted to one movie subtitle script. It is an English movie translated into Kurdish language. This study is confined to the investigation of one type of speech acts as classified by Searle namely commissive speech acts. In addition to that, the study focuses on the problematic commissive speech acts during translation from English into Kurdish, when there is loss of meaning.

\section{Data Analysis}

\subsection{Speech act and Illocutions}

In this section, the collected data will be presented in some tables, described in some detail and then analyzed. The main table shows the overall commissive speech acts which have translation problems, $i$.e. the speech acts identified in the selected movie (Pumpkinhead: Blood Feud). It can be seen that most commissive speech acts of the English scripts are compatible with the speech acts of Kurdish translation. Mistranslation ,sometimes, leads to the differences in speech act between the two languages. For example, in subtitle sample (8) commmissive became directive, Subtitle sample (9) commissive became expressive and Subtitle sample (14) commissive became representative and subtitle sample (20) commissive became declarative. It can ,also, be observed that commissive speech acts have certain illocutionary acts, such as promising , warning, and guaranteeing.

Table 1: Commissive Speech Acts in Pumpkinhead: Blood

Feud.

\begin{tabular}{|c|c|c|c|c|c|c|c|}
\hline$\overline{\text { No }}$ & $\begin{array}{l}\text { Movie Scripts } \\
\text { (Speech Act) }\end{array}$ & $\begin{array}{l}\text { Searle's Illocution } \\
\text { Categorary Act } \\
\text { y }\end{array}$ & $\begin{array}{l}\text { Movie scripts } \\
\text { in Kurdish }\end{array}$ & Error type & $\begin{array}{l}\text { English } \\
\text { rendering for } \\
\text { the Kurdish } \\
\text { subtitle } \\
\end{array}$ & $\begin{array}{l}\text { Searle } \\
\text { Category for } \\
\text { the Kurdish } \\
\text { translation } \\
\end{array}$ & $\begin{array}{l}\text { Illocutionary } \\
\text { act for Kurdish } \\
\text { translation }\end{array}$ \\
\hline & $\begin{array}{l}\text { You'll } \\
\text { see. } \underline{O h}, \\
\text { yeah, } \\
\text { you'll see }\end{array}$ & $\begin{array}{l}\text { Com Warni } \\
\mathrm{m} \text { ng }=\end{array}$ & دمبينى. بلكئي & DEL & $\begin{array}{l}\text { You will } \\
\text { see. Yes } \\
\text { you will } \\
\text { see }\end{array}$ & Comm & Warning $=$ \\
\hline & $\begin{array}{l}\text { It'll never } \\
\text { work out. }\end{array}$ & $\begin{array}{cc}\text { Com Guara } \\
\mathrm{m} \quad \text { nteein } \\
\mathrm{g}=\end{array}$ & 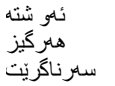 & ADD & $\begin{array}{l}\text { This thing } \\
\text { will never } \\
\text { work out }\end{array}$ & Comm & $\begin{array}{l}\text { Guaranteei } \\
\mathrm{ng}=\end{array}$ \\
\hline & $\begin{array}{l}\text { They will } \\
\text { pay for } \\
\text { this. I'll } \\
\text { get them } \\
\text { All of } \\
\text { them. } \\
\text { Everyone } \\
\text { of them }\end{array}$ & $\begin{array}{l}\text { Com Promis } \\
\mathrm{m} \quad \text { ing }=\end{array}$ & باجى نديَّ شنوانه & MIS & $\begin{array}{l}\text { They must } \\
\text { pay for that } \\
\text { I will } \\
\text { catch them } \\
\text { all one by } \\
\text { one }\end{array}$ & Comm & $\begin{array}{l}\text { Promising } \\
=\end{array}$ \\
\hline & $\begin{array}{l}\text { The } \\
\text { demon } \\
\text { will do } \\
\text { your } \\
\text { bidding } \\
\text { till it's } \\
\text { done }\end{array}$ & $\begin{array}{l}\text { Com Promis } \\
\mathrm{m} \quad \text { ing }=\end{array}$ & هلمويان، ياكك & MIS & $\begin{array}{l}\text { The ghost } \\
\text { will do } \\
\text { your } \\
\text { request till } \\
\text { it's done }\end{array}$ & Comm & $\begin{array}{l}\text { Promising } \\
=\end{array}$ \\
\hline & $\begin{array}{l}\text { And then } \\
\text { you will } \\
\text { pay the } \\
\text { price }\end{array}$ & $\begin{array}{l}\text { Com Warni } \\
\mathrm{m} \quad \mathrm{ng}=\end{array}$ & & MIS & $\begin{array}{l}\text { And that } \\
\text { time you } \\
\text { will pay } \\
\text { the price }\end{array}$ & Comm & $\begin{array}{l}\text { Warning } \\
=\end{array}$ \\
\hline & $\begin{array}{l}\text { If you do } \\
\text { this the } \\
\text { Hatfield } \\
\text { will pay a } \\
\text { terrible } \\
\text { price for } \\
\text { what they } \\
\text { have } \\
\text { done }\end{array}$ & $\begin{array}{l}\text { Com Promis } \\
\mathrm{m} \quad \text { ing }=\end{array}$ & داو ديّو مز مهكَّه & MIS & $\begin{array}{l}\text { If you do } \\
\text { this the } \\
\text { Hatfields } \\
\text { will lose. } \\
\text { They lose a } \\
\text { lot for } \\
\text { what they } \\
\text { have done }\end{array}$ & Comm & $\begin{array}{l}\text { Promising } \\
=\end{array}$ \\
\hline & $\begin{array}{l}\text { You'll } \\
\text { pay the } \\
\text { worst } \\
\text { price of } \\
\text { all }\end{array}$ & $\begin{array}{l}\text { Com Warni } \\
\mathrm{m} \quad \mathrm{ng}=\end{array}$ & تاندائو كاتهيتى & MIS & $\begin{array}{l}\text { You will } \\
\text { lose more } \\
\text { than } \\
\text { everyone }\end{array}$ & Comm & Warning $=$ \\
\hline & $\begin{array}{l}\text { It is } \\
\text { gonna } \\
\text { cost you } \\
\text { your very } \\
\text { soul, boy }\end{array}$ & $\begin{array}{l}\text { Com Warni } \\
\text { m ng \# }\end{array}$ & & MISDEL & $\begin{array}{l}\text { You have } \\
\text { to donate } \\
\text { your soul }\end{array}$ & Dirc & $\begin{array}{l}\text { Commandi } \\
\text { ng \# }\end{array}$ \\
\hline & $\begin{array}{l}\text { Your } \\
\text { revenge } \\
\text { will eat at }\end{array}$ & $\begin{array}{l}\text { Com Warni } \\
\mathrm{m} \quad \text { ng \# }\end{array}$ & 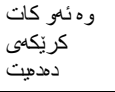 & $\begin{array}{l}\text { MIS } \\
\text { ADD } \\
\text { DEL }\end{array}$ & $\begin{array}{l}\text { Ah boy: } \\
\text { revenge } \\
\text { eats your }\end{array}$ & Expr & $\begin{array}{l}\text { Surprising } \\
\#\end{array}$ \\
\hline
\end{tabular}




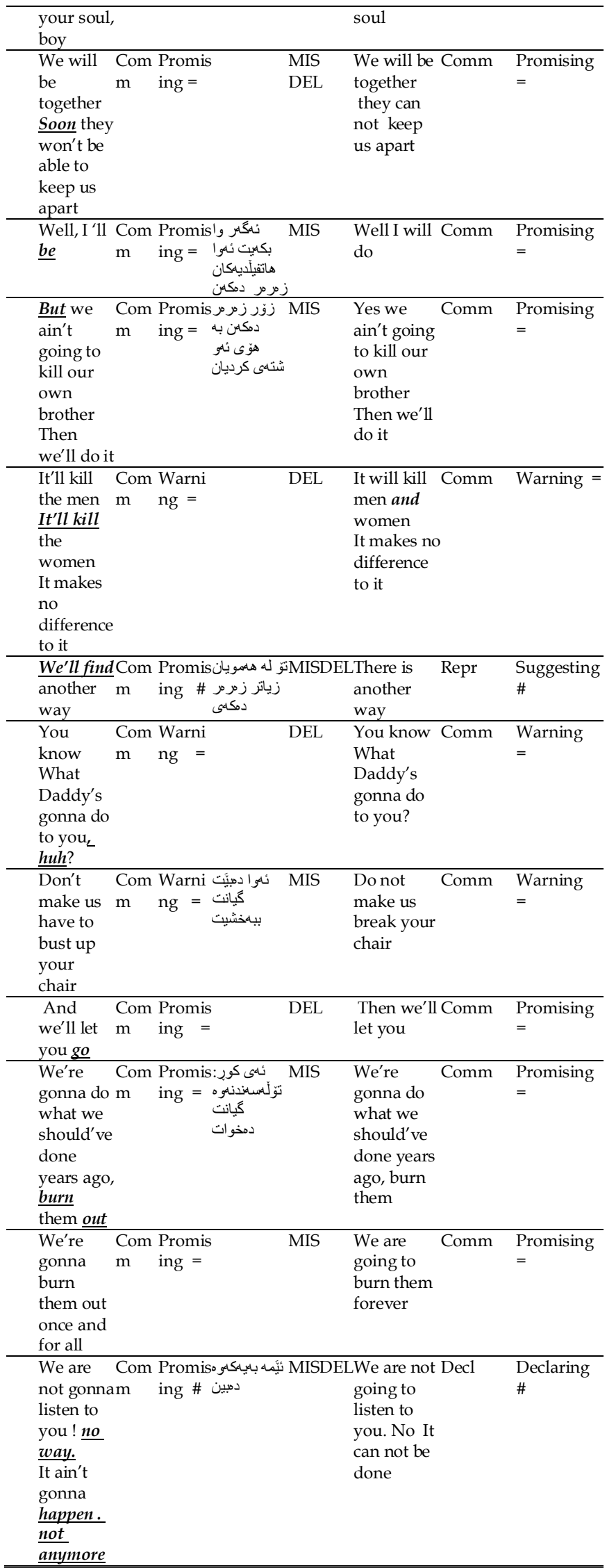

This table is divided into several sub tables for the sake of explaining and analyzing the data in more detail. Based on the illocutionary speech acts, this table is divided into three sub-tables promising, warning, and guaranteeing.

Table 2: illocutionary acts (promising) of commissive speech acts in Pumpkinhead: Blood Feud

\begin{tabular}{|c|c|c|c|c|c|c|c|c|}
\hline No & $\begin{array}{c}\text { Movie } \\
\text { Scripts } \\
\text { (Speech } \\
\text { Act) }\end{array}$ & $\begin{array}{l}\text { Searle's } \\
\text { Category }\end{array}$ & $\begin{array}{l}\text { Illocutionar } \\
\text { y Act }\end{array}$ & $\begin{array}{l}\text { Movie } \\
\text { scripts in } \\
\text { Kurdish }\end{array}$ & $\begin{array}{l}\text { Error } \\
\text { type }\end{array}$ & $\begin{array}{l}\text { English } \\
\text { renderin } \\
\text { g for the } \\
\text { Kurdish } \\
\text { subtitle }\end{array}$ & $\begin{array}{c}\text { Searle } \\
\text { Category } \\
\text { for the } \\
\text { Kurdish } \\
\text { translati } \\
\text { on }\end{array}$ & $\begin{array}{c}\text { Illocuti } \\
\text { onary } \\
\text { act for } \\
\text { Kurdis } \\
\text { h } \\
\text { i translat } \\
\text { ion } \\
\end{array}$ \\
\hline & $\begin{array}{l}\text { They will } \\
\text { pay for } \\
\text { this. I'll } \\
\text { get them } \\
\text { All of } \\
\text { them. } \\
\text { Everyone } \\
\text { of them }\end{array}$ & Comm & Promising $=$ & 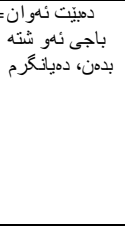 & MIS & $\begin{array}{l}\text { They } \\
\text { must pay } \\
\text { for that, } \\
\text { I will } \\
\text { catch } \\
\text { them all } \\
\text { one by } \\
\text { one }\end{array}$ & Comm & $\begin{array}{l}\text { Promisi } \\
\text { ng }=\end{array}$ \\
\hline & $\begin{array}{l}\text { The } \\
\text { demon } \\
\text { will do } \\
\text { your } \\
\text { bidding til } \\
\text { it's done }\end{array}$ & $\begin{array}{l}\text { Comm } \\
11\end{array}$ & Promising $=$ & يدك به هيكويان، & MIS & $\begin{array}{l}\text { The } \\
\text { ghost } \\
\text { will do } \\
\text { your } \\
\text { request } \\
\text { till it's } \\
\text { done }\end{array}$ & Comm & $\begin{array}{l}\text { Promisi } \\
\mathrm{ng}=\end{array}$ \\
\hline & $\begin{array}{l}\text { If you do } \\
\text { this the } \\
\text { Hatfield } \\
\text { will pay a } \\
\text { terrible } \\
\text { price for } \\
\text { what they } \\
\text { have done }\end{array}$ & Comm & Promising $=$ & & MIS & $\begin{array}{l}\text { If you do } \\
\text { this the } \\
\text { Hatfields } \\
\text { will lose. } \\
\text { They } \\
\text { lose a lot } \\
\text { for what } \\
\text { they } \\
\text { have } \\
\text { done }\end{array}$ & $\begin{array}{l}\text { Comm } \\
\text { s } \\
\text { t } \\
\\
\end{array}$ & $\begin{array}{l}\text { Promisi } \\
\text { ng }=\end{array}$ \\
\hline & $\begin{array}{l}\text { We will be } \\
\text { together } \\
\text { Soon they } \\
\text { won't be } \\
\text { able to } \\
\text { keep us } \\
\text { apart }\end{array}$ & Comm & Promising $=$ & 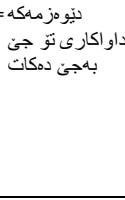 & $\begin{array}{l}\text { MIS } \\
\text { DEL }\end{array}$ & $\begin{array}{l}\text { We will } \\
\text { be } \\
\text { together } \\
\text { they can } \\
\text { not keep } \\
\text { us apart }\end{array}$ & Comm & $\begin{array}{l}\text { Promisi } \\
\text { ng }=\end{array}$ \\
\hline & $\begin{array}{l}\text { Well, I'll } \\
\underline{\underline{b e}}\end{array}$ & Comm & promising $=$ & 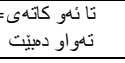 & MIS & $\begin{array}{l}\text { Well I } \\
\text { will do } \\
\end{array}$ & Comm & $\begin{array}{l}\text { Promisi } \\
\text { ng= }\end{array}$ \\
\hline & $\begin{array}{l}\text { But we } \\
\text { ain't going } \\
\text { to kill our } \\
\text { own } \\
\text { brother } \\
\text { Then we'll } \\
\text { do it }\end{array}$ & Comm & Promising $=$ & & MIS & $\begin{array}{l}\text { Yes we } \\
\text { ain't } \\
\text { going to } \\
\text { kill our } \\
\text { own } \\
\text { brother } \\
\text { Then } \\
\text { we'll do } \\
\text { it }\end{array}$ & Comm & $\begin{array}{l}\text { Promisi } \\
\text { ng }=\end{array}$ \\
\hline & $\begin{array}{l}\text { We'll find } \\
\text { another } \\
\text { way }\end{array}$ & Comm & $\begin{array}{l}\text { Promising } \\
\#\end{array}$ & 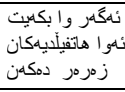 & $\begin{array}{l}\text { MIS } \\
\text { DEL }\end{array}$ & $\begin{array}{l}\text { There is } \\
\text { another } \\
\text { way }\end{array}$ & Repr & $\begin{array}{l}\text { Sugges } \\
\text { ting \# }\end{array}$ \\
\hline & $\begin{array}{l}\text { and we'll } \\
\text { let you go }\end{array}$ & Comm & $\begin{array}{l}\text { Promising } \\
=\end{array}$ & 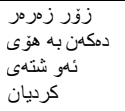 & DEL & $\begin{array}{l}\text { Then } \\
\text { we'll let } \\
\text { you }\end{array}$ & Comm & $\begin{array}{l}\text { Promisi } \\
\mathrm{ng}=\end{array}$ \\
\hline & $\begin{array}{l}\text { We're } \\
\text { gonna do } \\
\text { what we } \\
\text { should've } \\
\text { done year } \\
\text { ago, } \underline{\text { burn }} \\
\text { them } \underline{\text { out }}\end{array}$ & $\begin{array}{l}\text { Comm } \\
\mathrm{s}\end{array}$ & $\begin{array}{l}\text { promising } \\
=\end{array}$ & & MIS & $\begin{array}{l}\text { We're } \\
\text { gonna } \\
\text { do what } \\
\text { we } \\
\text { should'v } \\
\text { e done } \\
\text { years } \\
\text { ago, } \\
\text { burn } \\
\text { them }\end{array}$ & Comm & $\begin{array}{l}\overline{\text { Promisi }} \\
\mathrm{ng}=\end{array}$ \\
\hline & $\begin{array}{l}\text { We're } \\
\text { gonna } \\
\text { burn them } \\
\text { out once } \\
\text { and for all }\end{array}$ & Comm & $\begin{array}{l}\text { Promising } \\
=\end{array}$ & 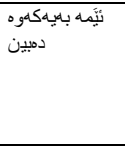 & MIS & $\begin{array}{l}\text { We are } \\
\text { going to } \\
\text { burn } \\
\text { them } \\
\text { forever }\end{array}$ & Comm & $\begin{array}{l}\text { Promisi } \\
\text { ng }=\end{array}$ \\
\hline & $\begin{array}{l}\text { We are no } \\
\text { gonna } \\
\text { listen to } \\
\text { you! no } \\
\text { way. } \\
\text { It ain't } \\
\text { gonna } \\
\text { happen. } \\
\underline{\text { not }} \\
\underline{\text { anymore }} \\
\end{array}$ & tComm & $\begin{array}{l}\text { Promising } \\
\#\end{array}$ & 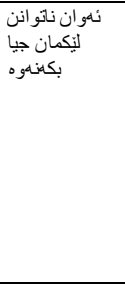 & $\begin{array}{l}\text { MIS } \\
\text { DEL }\end{array}$ & $\begin{array}{l}\text { We are } \\
\text { not } \\
\text { going to } \\
\text { listen to } \\
\text { you. No } \\
\text { It can not } \\
\text { be done }\end{array}$ & Decl & $\begin{array}{l}\text { Declari } \\
\text { ng \# }\end{array}$ \\
\hline
\end{tabular}

It can be observed from table (2) that in most of the 
cases, the English and Kurdish illocutions are the same.

However, (7-11) subtitle samples of the illocutionary speech act (promising) in the English version are not compatible with the illocutionary acts of those of the translated Kurdish version due to translation errors, such as in sample (7) where the illocutionary act (promising) became illocutionary act (suggesting) in the Kurdish subtitle. In sample (11) the illocution (promising) became (declaring) in the Kurdish subtitle.

Table 3: illocutionary (warning) of commissive speech acts in Pumpkinhead: Blood Feud

\begin{tabular}{|c|c|c|c|c|c|c|c|}
\hline No & $\begin{array}{l}\text { Movie } \\
\text { Scripts } \\
\text { (Speech } \\
\text { Act) }\end{array}$ & $\begin{array}{c}\text { Searle's } \\
\text { Category }\end{array}$ & $\begin{array}{c}\text { Movie } \\
\text { Illocutio } \\
\text { nary Act } \\
\text { scripts in } \\
\text { Kurdish }\end{array}$ & $\begin{array}{l}\text { Error } \\
\text { type }\end{array}$ & $\begin{array}{l}\text { English } \\
\text { rendering } \\
\text { for the } \\
\text { Kurdish } \\
\text { subtitle }\end{array}$ & $\begin{array}{c}\text { Searle } \\
\text { Catego } \\
\text { ry for } \\
\text { the } \\
\text { Kurdis } \\
\mathrm{h} \\
\text { translat } \\
\text { ion }\end{array}$ & $\begin{array}{l}\text { Illocuti } \\
\text { onary } \\
\text { act for } \\
\text { Kurdis } \\
\mathrm{h} \\
\text { translat } \\
\text { ion }\end{array}$ \\
\hline & $\begin{array}{l}\text { You'll see. } \\
\text { Oh, yeah, } \\
\text { you'll see }\end{array}$ & Comm & 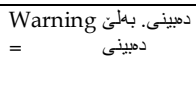 & DEL & $\begin{array}{l}\text { You will } \\
\text { see. Yes you } \\
\text { will see }\end{array}$ & Comm & $\begin{array}{l}\text { Warnin } \\
\mathrm{g}=\end{array}$ \\
\hline & $\begin{array}{l}\text { And then } \\
\text { you will } \\
\text { pay the } \\
\text { price }\end{array}$ & Comm & $\begin{array}{l}\text { Warning وه كرئكهى كات }= \\
= \\
=\end{array}$ & MIS & $\begin{array}{l}\text { And that } \\
\text { time you } \\
\text { will pay the } \\
\text { price }\end{array}$ & Comm & $\begin{array}{l}\text { Warnin } \\
\mathrm{g}=\end{array}$ \\
\hline & $\begin{array}{l}\text { You'll pay } \\
\text { the worst } \\
\text { price of all }\end{array}$ & Comm & $\begin{array}{l}\text { Warning } \\
=\end{array}$ & MIS & $\begin{array}{l}\text { You will } \\
\text { lose more } \\
\text { than } \\
\text { everyone }\end{array}$ & Comm & $\begin{array}{l}\text { Warnin } \\
\mathrm{g}=\end{array}$ \\
\hline & $\begin{array}{l}\text { It is gonna } \\
\text { cost you } \\
\text { your very } \\
\text { soul, boy }\end{array}$ & Comm & 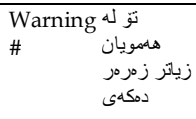 & MIS DEL & $\begin{array}{l}\text { You have to } \\
\text { donate your } \\
\text { soul }\end{array}$ & Dirc & $\begin{array}{l}\text { Comm } \\
\text { anding } \\
\#\end{array}$ \\
\hline & $\begin{array}{l}\text { Your } \\
\text { revenge wil } \\
\text { eat at your } \\
\text { soul, boy }\end{array}$ & Comm & $\begin{array}{l}\text { Warning } \\
\#\end{array}$ & $\begin{array}{l}\text { MIS } \\
\text { ADD } \\
\text { DEL }\end{array}$ & $\begin{array}{l}\text { Ah boy: } \\
\text { revenge } \\
\text { eats your } \\
\text { soul }\end{array}$ & Expr & $\begin{array}{l}\text { Surpris } \\
\text { ing \# }\end{array}$ \\
\hline & $\begin{array}{l}\text { It'll kill the } \\
\text { men } \\
\underline{\text { It'll kill the }} \\
\text { women } \\
\text { It makes no } \\
\text { difference } \\
\text { to it }\end{array}$ & Comm & 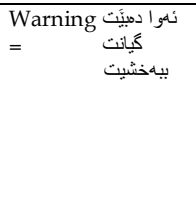 & DEL & $\begin{array}{l}\text { It will kill } \\
\text { men and } \\
\text { women } \\
\text { It makes no } \\
\text { difference } \\
\text { to it }\end{array}$ & Comm & $\begin{array}{l}\text { Warnin } \\
\mathrm{g}=\end{array}$ \\
\hline & $\begin{array}{l}\text { You know } \\
\text { What } \\
\text { Daddy's } \\
\text { gonna do to } \\
\text { you, huh? }\end{array}$ & Comm & $\begin{array}{l}\text { Warning } \\
=\end{array}$ & DEL & $\begin{array}{l}\text { You know } \\
\text { What } \\
\text { Daddy's } \\
\text { gonna do to } \\
\text { you? }\end{array}$ & Comm & $\begin{array}{l}\text { Warnin } \\
\mathrm{g}=\end{array}$ \\
\hline & $\begin{array}{l}\text { Don't make } \\
\text { us have to } \\
\text { bust up } \\
\text { your chair }\end{array}$ & Comm & 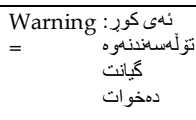 & MIS & $\begin{array}{l}\text { Do not } \\
\text { make us } \\
\text { break your } \\
\text { chair }\end{array}$ & Comm & $\begin{array}{l}\text { Warnin } \\
\mathrm{g}=\end{array}$ \\
\hline
\end{tabular}

It can be observed from the above table that in most cases, the illocutionary act (warning ) in the English and Kurdish language is identical. However, in subtitle sample (4) the illocutionary act (warning) in the English subtitle became illocutionary act of (commanding) in the Kurdish subtitle, subtitle sample (5) the illocutionary act of (warning) in English subtitle became illocutionary act of (surprising ) in the Kurdish subtitle. These differences between illocutionary acts of both languages are due to the influence of their mistranslation.

Table 4: illocutionary (guaranteeing) of commissive speech acts in Pumpkinhead: Blood Feud.

\begin{tabular}{|c|c|c|c|c|c|c|c|c|}
\hline No & $\begin{array}{c}\text { Movie } \\
\text { Scripts } \\
\text { (Speech } \\
\text { Act) }\end{array}$ & $\begin{array}{l}\text { Searle's } \\
\text { Category }\end{array}$ & $\begin{array}{l}\text { Illocutiona } \\
\text { ry Act }\end{array}$ & $\begin{array}{l}\text { a Movie scripts } \\
\text { in Kurdish }\end{array}$ & Error type & $\begin{array}{l}\text { English } \\
\text { rendering } \\
\text { for the } \\
\text { Kurdish } \\
\text { subtitle }\end{array}$ & $\begin{array}{l}\text { Searle } \\
\text { Catego } \\
\text { y for th } \\
\text { Kurdist } \\
\text { translat }\end{array}$ & $\begin{array}{l}\text { Illocutio } \\
\text { on nary act } \\
\text { e for } \\
\text { h Kurdish } \\
\text { ti translati }\end{array}$ \\
\hline 1 & $\begin{array}{l}\text { You'll } \\
\text { see. Oh, } \\
\text { yeah, } \\
\text { you'll see }\end{array}$ & Comm & $\begin{array}{l}\text { Warning } \\
=\end{array}$ & دمبينى. بهلىئ & DEL & $\begin{array}{l}\text { You will } \\
\text { see. Yes } \\
\text { you will } \\
\text { see }\end{array}$ & $\begin{array}{c}\text { on } \\
\text { Comm }\end{array}$ & \begin{tabular}{l}
\multicolumn{1}{c}{ on } \\
Warnin \\
$\mathrm{g}=$
\end{tabular} \\
\hline 2 & $\begin{array}{l}\text { It is } \\
\text { gonna } \\
\text { cost you } \\
\text { your very } \\
\text { soul, boy }\end{array}$ & Comm & Warning \# & 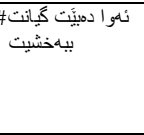 & MIS DEL & $\begin{array}{l}\text { You have } \\
\text { to donate } \\
\text { your soul }\end{array}$ & Dirc & $\begin{array}{l}\text { Comma } \\
\text { nding \# }\end{array}$ \\
\hline 3 & $\begin{array}{l}\text { We will } \\
\text { be } \\
\text { together } \\
\text { Soon they } \\
\text { won't be } \\
\text { able to } \\
\text { keep us } \\
\text { apart }\end{array}$ & $\begin{array}{l}\text { Comm } \\
\text { y }\end{array}$ & $\begin{array}{l}\text { Promising } \\
=\end{array}$ & 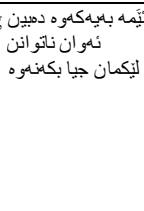 & MIS DEL & $\begin{array}{l}\text { We will be } \\
\text { together } \\
\text { they can } \\
\text { not keep us } \\
\text { apart }\end{array}$ & $\begin{array}{l}\text { Comm } \\
\text { s }\end{array}$ & $\begin{array}{l}\text { Promisi } \\
\mathrm{ng}=\end{array}$ \\
\hline 4 & $\begin{array}{l}\text { It'll kill } \\
\text { the men } \\
\text { It'll kill } \\
\text { the } \\
\text { women } \\
\text { It makes } \\
\text { no } \\
\text { difference } \\
\text { to it }\end{array}$ & Comm & $\begin{array}{l}\text { Warning } \\
=\end{array}$ & 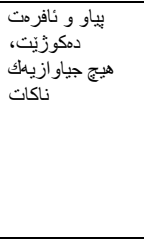 & DEL & $\begin{array}{l}\text { It will kill } \\
\text { men and } \\
\text { women } \\
\text { It makes no } \\
\text { difference } \\
\text { to it }\end{array}$ & Comm & $\begin{array}{l}\text { Warnin } \\
\mathrm{g}=\end{array}$ \\
\hline 5 & $\begin{array}{l}\text { We'll find } \\
\text { another } \\
\text { way }\end{array}$ & dComm & $\begin{array}{l}\text { Promising } \\
\#\end{array}$ & ريخهيةكى تر هليه ه: & MIS DEL & $\begin{array}{l}\text { There is } \\
\text { another } \\
\text { way }\end{array}$ & Repr & $\begin{array}{l}\text { Suggesti } \\
\text { ng \# }\end{array}$ \\
\hline 6 & $\begin{array}{l}\text { You } \\
\text { know } \\
\text { What } \\
\text { Daddy's } \\
\text { gonna do } \\
\text { to you, } \\
\text { huh? }\end{array}$ & Comm & $\begin{array}{l}\text { Warning } \\
=\end{array}$ & 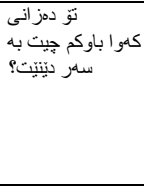 & DEL & $\begin{array}{l}\text { You know } \\
\text { What } \\
\text { Daddy's } \\
\text { gonna do to } \\
\text { you? }\end{array}$ & $\begin{array}{l}\text { Comm } \\
\text { o }\end{array}$ & $\begin{array}{l}\text { Warnin } \\
\mathrm{g}=\end{array}$ \\
\hline$\overline{7}$ & $\begin{array}{l}\text { And } \\
\text { we'll let } \\
\text { you go }\end{array}$ & Comm & $\begin{array}{l}\text { Promising } \\
=\end{array}$ & ئينجا وازهت لئَ & DEL & $\begin{array}{l}\text { Then we'll } \\
\text { let you }\end{array}$ & 1Comm & $\begin{array}{l}\text { Promisi } \\
\mathrm{ng}=\end{array}$ \\
\hline 8 & $\begin{array}{l}\text { We are } \\
\text { not gonna } \\
\text { listen to } \\
\text { you ! no } \\
\text { way. } \\
\text { It ain't } \\
\text { gonna } \\
\text { happen . } \\
\text { not } \\
\text { anymore }\end{array}$ & Comm & $\begin{array}{l}\text { Promising } \\
\#\end{array}$ & كَوَّ له تو ناكرين. & MIS DEL & $\begin{array}{l}\text { We are not } \\
\text { going to } \\
\text { listen to } \\
\text { you. It can } \\
\text { not be done }\end{array}$ & Decl & $\begin{array}{l}\text { Declarin } \\
\mathrm{g} \#\end{array}$ \\
\hline 9 & $\begin{array}{l}\text { Your } \\
\text { revenge } \\
\text { will eat at } \\
\text { your soul, } \\
\text { boy }\end{array}$ & Comm & $\begin{array}{l}\text { Warning } \\
\#\end{array}$ & 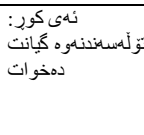 & $\begin{array}{l}\text { MIS ADD } \\
\text { توEL }\end{array}$ & $\begin{array}{l}\text { Ah boy: } \\
\text { revenge } \\
\text { eats your } \\
\text { soul }\end{array}$ & Expr & $\begin{array}{l}\text { Surprisi } \\
\text { ng \# }\end{array}$ \\
\hline
\end{tabular}

It can be observed from the above table that there is only one subtitle sample in which the illocutionary speech act (guaranteeing) in the English version is identical with the illocutionary speech act of the translated Kurdish version.

\subsection{Translation error}

The overall commissive speech act which had error in 
their translation were (20) samples. The focus will be on deletion errors which include omission of any item that leads to loss of meaning of the original message, i.e. when there is meaning loss. As it can be observed from the following tables, this study merely highlights deletion errors as it aims at meaning loss.

Table 5: Deletion errors of Commissive speech acts in Pumpkinhead: Blood Feud.

\begin{tabular}{|c|c|c|c|c|c|c|c|}
\hline No & $\begin{array}{l}\text { Movie } \\
\text { Scripts } \\
\text { (Speech Act) }\end{array}$ & $\begin{array}{l}\text { Searle Illocution } \\
\text { 's ary Act } \\
\text { Categ } \\
\text { ory }\end{array}$ & $\begin{array}{l}\text { Movie scripts in } \\
\text { Kurdish }\end{array}$ & $\begin{array}{l}\text { Error } \\
\text { type }\end{array}$ & $\begin{array}{l}\text { English } \\
\text { rendering for } \\
\text { the Kurdish } \\
\text { subtitle }\end{array}$ & $\begin{array}{l}\text { Searle } \\
\text { Categor } \\
\text { y for } \\
\text { the } \\
\text { Kurdish } \\
\text { translati } \\
\text { on } \\
\end{array}$ & $\begin{array}{l}\text { Illocutionar } \\
\text { y act for } \\
\text { Kurdish } \\
\text { translation } \\
\text { i }\end{array}$ \\
\hline 1 & $\begin{array}{l}\text { You'll see. } \\
\text { Oh, yeah, } \\
\text { you'll see }\end{array}$ & $\begin{array}{c}\text { Comm Warning } \\
=\end{array}$ & دميينى. بهلىي دمبينى & DEL & $\begin{array}{l}\text { You will } \\
\text { see. Yes you } \\
\text { will see }\end{array}$ & Comm & Warning $=$ \\
\hline 2 & $\begin{array}{l}\text { It is gonna } \\
\text { cost you your } \\
\text { very soul, } \\
\text { boy }\end{array}$ & r $\quad$ Comm Warning & 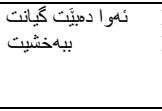 & $\begin{array}{l}\text { MIS } \\
\text { DEL }\end{array}$ & $\begin{array}{l}\text { You have to } \\
\text { donate your } \\
\text { soul }\end{array}$ & Dirc & $\begin{array}{l}\text { Commandi } \\
\text { ng \# }\end{array}$ \\
\hline 3 & $\begin{array}{l}\text { We will be } \\
\text { together } \\
\text { Soon they } \\
\text { won't be able } \\
\text { to keep us } \\
\text { apart }\end{array}$ & $\begin{array}{l}\text { Comm Promising } \\
=\end{array}$ & 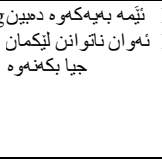 & $\begin{array}{l}\text { MIS } \\
\text { DEL }\end{array}$ & $\begin{array}{l}\text { We will be } \\
\text { together } \\
\text { they can not } \\
\text { keep us apart }\end{array}$ & Comm & $\begin{array}{l}\text { Promising } \\
=\end{array}$ \\
\hline 4 & $\begin{array}{l}\text { It'll kill the } \\
\text { men } \\
\text { It'll kill the } \\
\text { women } \\
\text { It makes no } \\
\text { difference to } \\
\text { it }\end{array}$ & $\begin{array}{c}\text { Comm Warning } \\
=\end{array}$ & 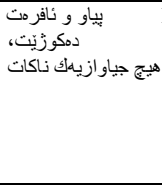 & DEL & $\begin{array}{l}\text { It will kill } \\
\text { men and } \\
\text { women } \\
\text { It makes no } \\
\text { difference to } \\
\text { it }\end{array}$ & Comm & Warning $=$ \\
\hline 5 & $\begin{array}{l}\text { We'll find } \\
\text { another way }\end{array}$ & $\begin{array}{c}\text { Comm Promising } \\
\# \\
\end{array}$ & ريكايهكى تر هليه و & $\begin{array}{l}\text { MIS } \\
\text { DEL }\end{array}$ & $\begin{array}{l}\text { There is } \\
\text { another way }\end{array}$ & Repr & $\begin{array}{l}\text { Suggesting } \\
\#\end{array}$ \\
\hline$\overline{6}$ & $\begin{array}{l}\text { You know } \\
\text { What } \\
\text { Daddy's } \\
\text { gonna do to } \\
\text { you, huh? }\end{array}$ & $\begin{array}{c}\text { Comm Warning } \\
=\end{array}$ & 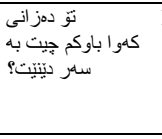 & DEL & $\begin{array}{l}\text { You know } \\
\text { What } \\
\text { Daddy's } \\
\text { gonna do to } \\
\text { you? }\end{array}$ & Comm & $\begin{array}{l}\text { Warning } \\
=\end{array}$ \\
\hline 7 & $\begin{array}{l}\text { And we'll let } \\
\text { you go }\end{array}$ & $\begin{array}{c}\text { etComm Promising } \\
=\end{array}$ & ائينجا وازت لدئَ & DEL & $\begin{array}{l}\text { Then we'll } \\
\text { let you }\end{array}$ & Comm & $\begin{array}{l}\text { Promising } \\
=\end{array}$ \\
\hline 8 & $\begin{array}{l}\text { We are not } \\
\text { gonna listen } \\
\text { to you ! no } \\
\text { way. } \\
\text { It ain't gonna } \\
\text { happen . not } \\
\text { anymore }\end{array}$ & $\begin{array}{l}\text { Comm Promising } \\
\# \\
\end{array}$ & 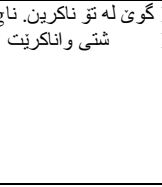 & $\begin{array}{l}\text { MIS } \\
\text { DEL }\end{array}$ & $\begin{array}{l}\text { We are not } \\
\text { going to } \\
\text { listen to you. } \\
\text { It can not be } \\
\text { done }\end{array}$ & Decl & $\begin{array}{l}\text { Declaring } \\
\#\end{array}$ \\
\hline 9 & $\begin{array}{l}\text { Your revenge } \\
\text { will eat at } \\
\text { your soul, } \\
\text { boy }\end{array}$ & $\begin{array}{c}\text { eComm Warning : } \\
\#\end{array}$ & 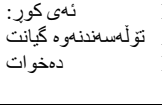 & $\begin{array}{l}\text { MIS } \\
\text { ADD } \\
\text { DEL }\end{array}$ & $\begin{array}{l}\text { Ah boy: } \\
\text { revenge eats } \\
\text { your soul }\end{array}$ & Expr & $\begin{array}{l}\text { Surprising } \\
\#\end{array}$ \\
\hline
\end{tabular}

It is observed from table (5) that exclamations have been deleted in subtitle samples (1) 'oh' \& (6)' $h u h h^{\prime}$. The translator faced difficulty in finding an equivalent exclamation in Kurdish language. Sometimes, underestimating an exclamation or neglecting it leads to this kind of deletion. The proper noun has also been deleted in subtitle sample (2) 'boy', The literature also supports such type of deletion, Chiu et al. (1999) state that the deletion of the first and middle name is acceptable when they are mentioned for the second time. The adjective has been deleted in subtitle sample (2) 'very' . It is ,also, observed from subtitle samples (3),
(8) 'soon', 'no way', 'not', 'anymore' that the adverbs have been deleted. One of the possibilities behind the deletion might be that the translator thinks deletion of adverbs will not affect the message or it might be due to the negligence or lack of attention.

It is evident that even pronouns have been deleted in the subtitle samples, 'it' (4), 'we' (5), 'your' (9). It is apparent that pronouns might be an easy aspect for translators but their deletion might be due to such reasons as carelessness, poor revision, and/or poor decision making as to their importance. In addition to that, the modal verb has also been deleted in subtitle samples (4), (5) 'will'. The modal verb has been repeated twice in subtitle (4). That is why the translator has an excuse for deleting the second modal and using ellipsis. In subtitle samples $(5,7,4)$, the main verbs 'find', 'go', 'kill' have been deleted by the translator, while the verb 'kill' has been repeated twice in the same subtitle. That is why the translator deleted them and used ellipsis . The exclamation mark '!' has also been deleted in sample (8). These deletions might be due to lack of revision or attention or negligence or not finding an equivalent word or expression in Kurdish language.

Table 6: Deletion errors of promising illocutions of speech acts in Pumpkinhead: Blood Feud.

\begin{tabular}{|c|c|c|c|c|c|c|c|c|}
\hline No & $\begin{array}{c}\text { Movie } \\
\text { Scripts } \\
\text { (Speech } \\
\text { Act) }\end{array}$ & $\begin{array}{c}\text { Searle's } \\
\text { Categor } \\
y\end{array}$ & $\begin{array}{l}\text { sillocutio } \\
\text { r nary } \\
\text { Act }\end{array}$ & $\begin{array}{l}\text { Movie } \\
\text { scripts in } \\
\text { Kurdish }\end{array}$ & $\begin{array}{l}\text { Error } \\
\text { type }\end{array}$ & $\begin{array}{c}\text { English } \\
\text { rendering } \\
\text { for the } \\
\text { Kurdish } \\
\text { subtitle }\end{array}$ & $\begin{array}{c}\text { Searle } \\
\text { Category for } \\
\text { the Kurdish } \\
\text { translation }\end{array}$ & $\begin{array}{l}\text { Illocutionary } \\
\text { act for } \\
\text { Kurdish } \\
\text { translation }\end{array}$ \\
\hline 1 & $\begin{array}{l}\text { We will be } \\
\text { together } \\
\text { Soon they } \\
\text { won't be } \\
\text { able to } \\
\text { keep us } \\
\text { apart }\end{array}$ & Comm & $\begin{array}{l}\text { Promisi } \\
\mathrm{ng}=\end{array}$ & بلديمكانوهُ & $\begin{array}{l}\text { MIS } \\
\text { DEL }\end{array}$ & $\begin{array}{l}\text { We will } \\
\text { be } \\
\text { together } \\
\text { they can } \\
\text { not keep } \\
\text { us apart }\end{array}$ & Comm & Promising $=$ \\
\hline 2 & $\begin{array}{l}\text { We'll find } \\
\text { another } \\
\text { way }\end{array}$ & Comm & $\begin{array}{l}\text { Promisi } \\
\text { ng \# }\end{array}$ & 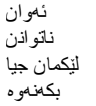 & $\begin{array}{l}\text { MIS } \\
\text { DEL }\end{array}$ & $\begin{array}{l}\text { There is } \\
\text { another } \\
\text { way }\end{array}$ & Repr & Suggesting \# \\
\hline 3 & $\begin{array}{l}\text { And we'll } \\
\text { let you go }\end{array}$ & Comm & $\begin{array}{l}\text { Promisi } \\
\mathrm{ng}=\end{array}$ & & DEL & $\begin{array}{l}\text { Then } \\
\text { we'll let } \\
\text { you }\end{array}$ & Comm & Promising $=$ \\
\hline 4 & $\begin{array}{l}\text { We are not } \\
\text { gonna } \\
\text { listen to } \\
\text { you ! no } \\
\text { way. } \\
\text { It ain't } \\
\text { gonna } \\
\text { happen . } \\
\text { not } \\
\text { anymore }\end{array}$ & Comm & $\begin{array}{l}\text { Promisi } \\
\text { ng \# }\end{array}$ & تريخميكى هليه & $\begin{array}{l}\text { MIS } \\
\text { DEL }\end{array}$ & $\begin{array}{l}\text { We are } \\
\text { not going } \\
\text { to listen } \\
\text { to you. It } \\
\text { can not } \\
\text { be done }\end{array}$ & Decl & Declaring \# \\
\hline
\end{tabular}

As it can be noticed from table (6), there are 4 samples 
of deletion error out of 9 deletion error when it comes to translating promising illocutions. The deleted items are the adverbs 'soon', 'no way', 'not', 'anymore'. The

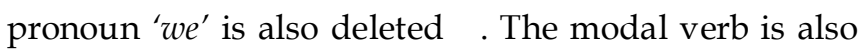
deleted 'will'. The main verb is also deleted ' $g o$ '. It is evident that adverbs are the most common deletion error of promising illocutions while pronouns, modal verbs, main verbs and exclamation marks are the least common deletion errors in promising illocutions.

Table (7) Deletion errors of warning illocutions of speech acts in Pumpkinhead: Blood Feud.

\begin{tabular}{|c|c|c|c|c|c|c|c|c|}
\hline No & $\begin{array}{l}\text { Movie Scripts } \\
\text { (Speech Act) }\end{array}$ & $\begin{array}{l}\text { Searle's } \\
\text { Category }\end{array}$ & $\begin{array}{l}\text { Illocution } \\
\text { ary Act }\end{array}$ & $\begin{array}{c}\text { Movie } \\
\text { scripts in } \\
\text { Kurdish }\end{array}$ & $\begin{array}{l}\text { Error } \\
\text { type }\end{array}$ & $\begin{array}{c}\text { English } \\
\text { rendering } \\
\text { for the } \\
\text { Kurdish } \\
\text { subtitle }\end{array}$ & $\begin{array}{c}\text { Searle } \\
\text { Categor } \\
\text { y for the } \\
\text { Kurdish } \\
\text { translati } \\
\text { on }\end{array}$ & $\begin{array}{l}\text { Illocution } \\
\text { ary act for } \\
\text { Kurdish } \\
\text { i translatio } \\
n\end{array}$ \\
\hline 1 & $\begin{array}{l}\text { You'll see. Oh, } \\
\text { yeah, you'll see }\end{array}$ & Comm & $\begin{array}{l}\text { Warning } \\
=\end{array}$ & دمبينى. بهلىي & DEL & $\begin{array}{l}\text { You will } \\
\text { see. Yes } \\
\text { you will see }\end{array}$ & Comm & $\begin{array}{l}\text { Warning } \\
=\end{array}$ \\
\hline 2 & $\begin{array}{l}\text { It is gonna cost } \\
\text { you your very } \\
\text { soul, boy }\end{array}$ & Comm & $\begin{array}{l}\text { Warning } \\
\#\end{array}$ & بئما كيانت & $\begin{array}{l}\text { MIS } \\
\text { DEL }\end{array}$ & $\begin{array}{l}\text { You have } \\
\text { to donate } \\
\text { your soul }\end{array}$ & Dirc & $\begin{array}{l}\text { Comman } \\
\text { ding \# }\end{array}$ \\
\hline 3 & $\begin{array}{l}\text { It'll kill the men } \\
\text { It'll kill the women } \\
\text { It makes no } \\
\text { difference to it }\end{array}$ & Comm & $\begin{array}{l}\text { Warning } \\
=\end{array}$ & & DEL & $\begin{array}{l}\text { It will kill } \\
\text { men and } \\
\text { women } \\
\text { It makes no } \\
\text { difference } \\
\text { to it }\end{array}$ & Comm & $\begin{array}{l}\text { Warning } \\
=\end{array}$ \\
\hline 4 & $\begin{array}{l}\text { You know } \\
\text { What Daddy's } \\
\text { gonna do to you, } \\
\text { huh? }\end{array}$ & Comm & $\begin{array}{l}\text { Warning } \\
=\end{array}$ & 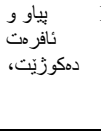 & DEL & $\begin{array}{l}\text { You know } \\
\text { What } \\
\text { Daddy's } \\
\text { gonna do to } \\
\text { you? }\end{array}$ & Comm & $\begin{array}{l}\text { Warning } \\
=\end{array}$ \\
\hline 5 & $\begin{array}{l}\text { Your revenge will } \\
\text { eat at your soul, } \\
\text { boy }\end{array}$ & Comm & Warning & جياو ازياتك هيج & $\begin{array}{l}\text { MIS } \\
\text { ADD } \\
\text { DEL }\end{array}$ & $\begin{array}{l}\text { Ah boy: } \\
\text { revenge } \\
\text { eats your } \\
\text { soul }\end{array}$ & Expr & $\begin{array}{l}\text { Surprisin } \\
\mathrm{g} \#\end{array}$ \\
\hline
\end{tabular}

As it can be noticed from table (7), there are 5 samples of deletion error out of 9 deletion error when it comes to translating warning illocutions. The deleted exclamations are 'oh', 'huh'. The pronouns 'your', 'it' are also deleted . The modal verb'will' is also deleted. The main verb 'kill' is also deleted. The adjective 'very' is also deleted. It is evident that, the exclamation word and pronoun are the most common deletion errors of warning illocutions while the modal verb, main verb and adjective are the least common deletion errors in warning illocutions.

\section{Conclusion}

It can be concluded that most speech acts and their illocutions in English language are compatible with illocutions of the Kurdish translation. However, in some cases, there is no compatibility between speech acts and their illocutions in the two languages. This may be due to the wide gap between the original text and the target text, in terms of rendering a commissive speech act into a directive, expressive, and a representative one. Moreover, the illocutions of promising and warning have become suggesting, commanding, and surprising.

It has also been revealed that the majority of the deleted items belong to promising and warning illocutions. Meaning losses represented by nouns, verbs, adverbs, adjectives, pronouns, modal verbs, exclamation marks, and other words are of various types. But adverbs are the most common type of deletion errors. Verbs and pronouns come second on the list of deletion errors. Modal verb and exclamation words come in the third place followed by nouns and adjectives and exclamation marks which are the least common type of deletion. All these losses are expected to be due to translation errors committed by the translator.

\section{References}

1. Abdulwahab, M.K. , Muhammad, H.A and Beneditte, M. (2020). Translating directive speech acts in movie subtitle from English into Kurdish. Zanco Journal Salahaddin.

2. Al-Sulaimaan, M. (2010). Semantics and pragmatics. Baghdad: Daar Ibn Al-Atheer.

3. Anderson, G. \& Arsenault, N. (1998). Fundamentals of educational research (2nd Edition). London: Falmer Press.

4. Colina, S. (2015). Fundamentals of translation. Cambridge: Cambridge University Press.

5. Chen, I. (1996). Lun zhong wen zh mu gan tan ci zhi fan yi. An unpublished MA thesis, Fu jen Catholic university Tiawan, ROC.

6. Chen, S. J. (2004). 'Linguistic dimensions of subtitling: perspectives from Taiwan'. Meta. 49 (1), pp. 115-124.

7. Crane, D. (2014). Cultural globalization and the dominance of the American film industry: cultural policies, national film industries, and transnational film. International journal of cultural policy. (20)4.

8. Fromkin, F., Rodman, R. and Hyams, N. (2003). An Introduction to language ( $7^{\text {th }}$ edition). Boston: Thomson.

9. Guerra, A. F. (2012). Translating culture: problems, strategies and practical realities. A Journal of Literature, Culture and Literary Translation. 1(12).

10. Ghazala, H. (2008). Translation As problems and solutions: A Textbook for University Students and Trainee Translators. Lebanon: Dar el-ilmlilmalayin.

11. Gotttlieb, H. (1998). Subtitling. In Mona Baker (Ed) 
Routledge Encyclopedia of Translation Studies (pp. 244248). London: Routledge.

12. Krippendorp, K. (2004). Content analysis: an introduction to its methodology. Thousand Oaks: Sage.

13. LoCastro, V. (2012). Pragmatics for language educators: A sociolinguistic perspective. New York: Routledge.

14. Leech, G. (1983). Principles of pragmatics. London: Longman.

15. Liu, M. (2003). Contemporary studies of translation. Beijing: China Translation \& Publishing Corporation.

16. Luyken, G.M., Thomas, H., Jo, L. B., Helene, R. And Hermans, S. (1991). Overcoming language barriers in television: dubbing and subtitling for the European audience. Manchester. European Institute for the Media.

17. Muhammad, H.A. (2017). Semantic loss in translating movie subtitles from English into Kurdish (Witch Hunter as a sample. Journal of Raparin University. 4 (11).

18. Mackey, A. \& Gass, S. M. (2005). Second language research; methodology and design. New Jersey: Routledge.

19. Munday, J. (2012). Introducing translation studies: theories and applications. London: Routledge.

20. Rao, S. (2007). The globalization of Bollywood: An ethnography of non-elite audiences in India. The Communication Review, Vol. 10, 57-76.

21. Sultan, K. M. (2007). The Semantic, pragmatics and translation of speech acts. Journal of College of Basic Education. (50)

22. Schreier, M. (2012). Qualitative content analysis in practice. London: Sage.

23. Yule, G. (1996). Pragmatics. Oxford: Oxford University Press. 